\title{
New Asymptotic Bounds on the Size of Multiple Packings of the Euclidean Sphere
}

\author{
Vladimir Blinovsky • Simon Litsyn
}

Received: 19 July 2010 / Revised: 9 February 2011 / Accepted: 17 February 2011 /

Published online: 11 March 2011

(C) Springer Science+Business Media, LLC 2011

\begin{abstract}
Using lower bounds on components of the distance spectrum of a code on the Euclidean sphere obtained by linear programming, we derive new, better than known, upper bounds on the size of multiple packings of spherical caps on the surface of the sphere.
\end{abstract}

Keywords Sphere packings $\cdot$ Multiple packings $\cdot$ Spherical codes

\section{Introduction}

Let $\mathbb{S}^{n-1}(\bar{x}, r) \subset \mathbb{R}^{n}$ be the sphere of radius $r$ centered at $\bar{x} \in \mathbb{R}^{n}$, and $\mathbb{B}^{n}(\bar{x}, r) \subset \mathbb{R}^{n}$ be the (closed) ball of radius $r$ centered at $\bar{x}$. Denote $\mathbb{S}^{n-1} \triangleq \mathbb{S}^{n-1}(\overline{0}, 1)$. We say that a (finite) set $\mathcal{K}^{n} \subset \mathbb{S}^{n-1}(\overline{0}, r)$ is a packing by balls or spherical caps of radius $t$ with multiplicity $L$ iff for any set of $(L+1)$ different points $\left\{\bar{x}_{1}, \ldots, \bar{x}_{L+1}\right\} \subset \mathcal{K}^{n}$, we have

$$
\bigcap_{i=1}^{L+1} B^{n}\left(\bar{x}_{i}, t\right)=\emptyset .
$$

The same condition can be written as

$$
\max _{\bar{x} \in \mathbb{R}^{n}}\left|B^{n}(\bar{x}, t) \cap \mathcal{K}^{n}\right| \leq L .
$$

V. Blinovsky

Institute of Information Transmission Problems, B. Karetnyi, 19, Moscow, Russia

e-mail: vblinovs@yandex.ru

S. Litsyn ( $₫)$

Electrical Engineering-Systems Department, Tel Aviv University, 69978 Tel Aviv, Israel

e-mail: litsyn@eng.tau.ac.il 
The set $\mathcal{K}^{n}$ is also a code guaranteeing list decoding within radius $t$ with the list size at most $L$.

Let $t_{L}\left(\mathcal{K}^{n}\right)$ be the supremum of $t$ with the above property. Next we consider a sequence of codes $\mathcal{K}^{n}, n=2,3, \ldots$, on the unit sphere and assume that $n \rightarrow \infty$ and $\ln \left|\mathcal{K}^{n}\right| \sim R n$ for some $R>0$. We call $R$ the code rate. Our goal is to obtain an asymptotic upper bound on

$$
t_{L}=\limsup _{n \rightarrow \infty} \sup _{\left|\mathcal{K}^{n}\right| \geq \exp (R n)} \frac{\sup t_{L}\left(\mathcal{K}^{n}\right)}{n} .
$$

The best previously known bound was first proved by Blachman and Few in [3], and later it was rederived in [4] using an alternative approach.

Theorem 1 The following inequality is valid:

$$
\begin{aligned}
R & \leq \frac{1}{2} \ln \frac{L}{(L+1) t_{L}^{2}}, \\
t_{L} & \leq\left(\frac{1}{2} \ln \frac{L}{(L+1) R}\right)^{1 / 2} .
\end{aligned}
$$

This is to be compared with the best currently known lower (existence) bound on $t_{L}$ which has been proved in [4]:

$$
R \geq \frac{1}{2} \ln \frac{L}{(L+1) t_{L}^{2}}+\frac{1}{2 L} \ln \frac{1}{(L+1)\left(1-t_{L}^{2}\right)} .
$$

In this paper we derive a new upper bound on $t_{L}$.

Theorem 2 The following inequality is valid:

$$
t_{L} \leq\left(\frac{1-L}{L}+\frac{1}{\min _{\phi \in\left(e^{-R}, 1\right)}\left(\phi \sin \frac{\theta_{\ell}(R+\ln \phi)}{2}\right)^{2}}\right)^{-1 / 2},
$$

where $\theta_{\ell}$ is the unique root of the equation

$$
\frac{1+\sin z}{2 \sin z} \cdot H\left(\frac{1-\sin z}{1+\sin z}\right)=R,
$$

and

$$
H(x)=-x \ln x-(1-x) \ln (1-x) .
$$

As we will see, this bound improves on (2).

The idea of the proof, presented in the next section, is as follows. We use an inequality derived in [2] that shows that in a spherical code of given size there exists a code vector having in its vicinity an essentially large number of neighboring code vectors. This is equivalent to saying that there exists a ball of dimension one less than 
the initial containing many code vectors. Applying this inequality recursively $L+1$ times, we conclude that there exist a ball containing at least $L+1$ code vectors. The radius of this ball can be easily translated into an upper bound on the radius of $L$ fold packing. Bounding the mentioned radius completes the derivation. Furthermore, using a simplification of the bound, we prove that, at least for low rates, it is better than the best earlier known bound (2).

\section{Proof of Theorem 2}

For a spherical code $\mathcal{K}$, we define its distance spectrum respective to a $\bar{c} \in \mathcal{K}$ as follows:

$$
b_{\mathcal{K}}(x, \bar{c})=\left\{\bar{c}_{1}: \bar{c}_{1} \in \mathcal{K},\left(\bar{c}, \bar{c}_{1}\right) /\left(\|\bar{c}\|\left\|\bar{c}_{1}\right\|\right)=x\right\},
$$

where $(\bar{a}, \bar{b})=a_{1} b_{1}+\cdots+a_{n} b_{n}$ stands for the scalar product. We will see that in any code of given size there exists a code vector possessing a big number of neighboring code vectors.

To state such a result, we will need some notation. For $\theta \leq 2 \varphi$, let

$$
\begin{aligned}
\alpha_{\varphi}(\theta)= & 2 \arcsin \frac{\sin (\theta / 2)}{\sin \varphi}, \\
\beta_{\varphi}(\theta)= & \arccos \frac{\cos \varphi}{\cos (\theta / 2)}, \\
j(x, y)= & (1+y) H\left(\frac{y}{1+y}\right) \\
& -\ln \left(\frac{1}{2}\left(x+\sqrt{(1+2 y)^{2} x^{2}-4 y(1+y)}\right)\right) \\
& +(1+2 y) \ln \frac{(1+2 y) x+\sqrt{(1+2 y)^{2} x^{2}-4 y(1+y)}}{2 y(1+y)} .
\end{aligned}
$$

For a given $R$, denote by $\rho_{\ell}$ the unique solution of the equation

$$
R=(1+\rho) H\left(\frac{\rho}{1+\rho}\right) \text {. }
$$

Notice that

$$
\frac{2 \sqrt{\rho_{\ell}\left(1+\rho_{\ell}\right)}}{1+2 \rho_{\ell}}=\cos \theta_{\ell},
$$

and if $\theta$ is the minimum angle between any pair of points from a code $\mathcal{K}^{n} \subset \mathbb{S}^{n-1}$, then the rate $R$ of this code satisfies the inequality [6]

$$
R \leq R(\theta)
$$

We will use the following result from [2] (see also [1] for a weaker result). 
Theorem 3 For $\mathcal{K}^{n} \subset \mathbb{S}^{n-1}(\overline{0}, r)$ with

$$
\ln \left|\mathcal{K}^{n}\right|=\operatorname{Rn}(1+o(1))
$$

and $\rho, \varphi$ satisfying

$$
e^{-R} \leq \sin \varphi \leq 1, \quad 0 \leq \rho \leq \rho_{\ell}(R+\ln \sin \varphi)
$$

there exists $\theta$ and $\bar{c} \in \mathcal{K}^{n}$ such that

$$
\frac{2 \sqrt{\rho(1+\rho)}}{1+2 \rho} \leq \cos \alpha_{\varphi}(\theta) .
$$

Moreover, after shifting the code vectors by $o(1), n \rightarrow \infty$, from their initial positions in such a way that they still belong to the sphere, we have

$$
\frac{1}{n} \ln b_{\mathcal{K}^{n}}(\cos \theta, \bar{c}) \geq R+2 \ln \sin \varphi-\ln \sin \beta \varphi(\theta)-j\left(\cos \alpha_{\varphi}(\theta), \rho\right)+o(1) .
$$

It is easy to see that $b_{\mathcal{K}^{n}}(\cos \theta, \bar{c})$ is the number of code vectors (after shifting) in the cross-section of the sphere by the hyperplane orthogonal to the vector $\bar{c}$ in the point $r \cos \theta \bar{c}$, where $r$ is the radius of the sphere.

Next, we describe a recursive procedure of constructing a simplex of $(L+1)$ codewords $\left\{\bar{c}_{0}, \ldots, \bar{c}_{L}\right\} \subset \mathcal{K}^{n}$ with sufficiently small pairwise distances and therefore yielding the existence of a ball $B^{n}(\bar{x}, r)$ of sufficiently small minimum radius $r$ containing all the points from the simplex.

We start with the code $\mathcal{K}_{0}^{n} \subset \mathbb{S}^{n-1}$ and consider the cross-section of $\mathbb{S}^{n-1}$ by a hyperplane. Then for some $\bar{c}_{0}$, the following is true: for each $\varphi_{1}, \rho_{1}$ as in Theorem 3 , there exists $\theta_{1}$, as in Theorem 3 , such that the cross-section of $\mathbb{S}^{n-1}$ by the hyperplane orthogonal to $\bar{c}_{0}$ and having the distance $\cos \theta_{1}$ from the origin contains (after shifting) the number of code vectors $b_{\mathcal{K}_{0}^{n}}\left(\cos \theta_{1}, \bar{c}_{0}\right)$ which satisfies inequality (6).

Further notice that the cross-section of the sphere by hyperplane is again a sphere of dimension one less. The new sphere has radius $r \sin \theta$ and is centered in $r \cos (\theta) \frac{\bar{c}_{0}}{\left\|\bar{c}_{0}\right\|}$ (given the initial sphere has radius $r$ ).

Next, we shift the center of this new sphere to the origin and once again consider the cross-section of this new sphere by hyperplane as at the previous step. Now we have the new code $\mathcal{K}_{1}^{n}$ of cardinality $b_{\mathcal{K}_{0}^{n}}\left(\cos \theta_{1}, \bar{c}_{0}\right)$, and the new cross-section contains (after shifting), for given

$$
\begin{gathered}
\arcsin \left(e^{-\ln b_{\mathcal{K}_{0}^{n}}\left(\cos \theta_{1}, \bar{c}_{0}\right) / n}\right) \leq \varphi_{2} \leq \pi / 2, \\
0 \leq \rho_{2} \leq \rho_{\ell}\left(\ln b_{\mathcal{K}_{0}^{n}}\left(\cos \theta_{1}, \bar{c}\right) / n+\ln \sin \varphi_{1}\right),
\end{gathered}
$$

at least

$$
b_{\mathcal{K}_{1}^{n}}\left(\cos \theta_{2}, \rho_{1}\right)
$$


code vectors from $\mathcal{K}_{1}^{n}$, where

$$
\begin{aligned}
\frac{\ln b_{\mathcal{K}_{1}^{n}}\left(\cos \theta_{2}, \bar{c}_{1}\right)}{n} \geq & \frac{\ln b_{\mathcal{K}_{0}^{n}}\left(\cos \theta_{1}, \bar{c}_{0}\right)}{n}+2 \ln \sin \varphi_{2}-\ln \sin \beta_{\varphi_{2}}\left(\theta_{2}\right) \\
& -j\left(\cos \alpha_{\varphi_{2}}\left(\theta_{2}\right), \rho_{2}\right)+o(1) .
\end{aligned}
$$

At this second step we choose new $\varphi_{2}, \rho_{2}, \theta_{2}, \bar{c}_{1}$.

This procedure can be continued further on. Let us provide a formal description of the procedure. At the 0 th step we have a code $\mathcal{K}_{0}^{n} \subset \mathbb{S}^{n-1}, \ln \left|\mathcal{K}_{0}^{n}\right| \sim n R_{0}\left(R_{0}=R\right)$. At the $i$ th step, $i \geq 1$, we obtain a code $\mathcal{K}_{i}^{n}$ of rate $\frac{1}{n} \ln \left|\mathcal{K}_{i}^{n}\right| \sim R_{i}$ such that

$$
R_{i} \geq R_{i-1}+2 \ln \sin \varphi_{i}-\ln \sin \beta_{\varphi_{i}}\left(\theta_{i}\right)-j\left(\cos \alpha_{\varphi_{i}}\left(\theta_{i}\right), \rho_{i}\right) .
$$

We undertake this action $(L+1)$ times, and at the $i$ th step, $i \geq 1$, we pick a new code vector $\bar{c}_{i}$ such that its distance from $\bar{c}_{j}, 0 \leq j<i$, is

$$
d_{j}=2 r_{j} \sin \frac{\theta_{j+1}}{2} .
$$

We stop when we fix $(L+1)$ code vectors $\bar{c}_{i} \in \mathcal{K}_{i}^{n}, i=0,1, \ldots, L$. Note also that $\mathcal{K}_{L}^{n} \subset \mathcal{K}_{L-1}^{n} \subset \cdots \subset \mathcal{K}_{0}^{n}$. Now we optimize the set $d_{j}, j=0,1, \ldots, L-1$, in such a way that the simplex on the vertices $\bar{c}_{i}$ is contained in a ball of minimum possible radius $t_{L}$ (the optimization is over $\varphi_{i}, \rho_{i}$, and at the same time assuming the worst case for the choice of $\theta_{i}$ ). This way we deduce the existence of a point in $\mathbb{R}^{n}$ covered by $(L+1)$ balls of radius $t_{L}$ with the centers in coding points, which yields that an arbitrary code of rate $R$ on the Euclidean sphere is an $L$-packing by balls of a radius strictly smaller than $t_{L}$.

We call the set $\left(\theta_{1}, \ldots, \theta_{L}\right)$ admissible if there exist sets $\varphi_{1}, \ldots, \varphi_{L} ; \rho_{1}, \ldots, \rho_{L}$, such that

$$
e^{-R_{i-1}} \leq \sin \varphi_{i}, \quad 0 \leq \rho_{i} \leq \rho_{\ell}\left(R_{i-1}+\ln \sin \varphi_{i-1}\right)
$$

and

$$
R_{i}=R_{i-1}+2 \ln \sin \varphi_{i}-\ln \sin \beta_{\varphi_{i}}\left(\theta_{i}\right)-j\left(\cos \alpha_{\varphi_{i}}\left(\theta_{i}\right), \rho_{i}\right),
$$

$i=1, \ldots, L$. We say that the set $\left(\varphi_{1}, \ldots, \varphi_{L}, \rho_{1}, \ldots, \rho_{L}\right)$ is admissible if there exists a set $\theta_{1}, \ldots, \theta_{L}$ which is admissible for these $\varphi_{i}, \rho_{i}$.

If $t\left(d_{0}, \ldots, d_{L-1}\right)$ is the minimum radius of a ball which contains the simplex $\left\{\bar{c}_{0}, \ldots, \bar{c}_{L}\right\}$ such that $\left\|\bar{c}_{i}-\bar{c}_{j}\right\|=d_{i}=2 r_{i} \sin \frac{\theta_{i+1}}{2}, i<j$, and $r_{i}=r_{i-1} \sin \theta_{i}, r_{0}=1$, then (see, for example, [5])

$$
t\left(d_{0}, \ldots, d_{L-1}\right)=\max _{I: \gamma^{I}\left(\Gamma^{I}\right)^{-1}\left(\gamma^{I}\right)^{T} \geq 0} \frac{1}{2} \sqrt{\gamma^{I}\left(\Gamma^{I}\right)^{-1}\left(\gamma^{I}\right)^{T}},
$$

where

$$
\gamma=\left(\gamma_{1}, \ldots, \gamma_{L}\right), \quad \Gamma=\left\|\Gamma_{i j}\right\|
$$


and

$$
\gamma_{i}=d_{0}^{2}, \quad \Gamma_{i, j}=\left(\bar{c}_{0}-\bar{c}_{i}, \bar{c}_{0}-\bar{c}_{j}\right) .
$$

Here $\gamma^{I}, \Gamma^{I}$ are the vector and matrix which are obtained from the initial vector $\gamma$ and matrix $\Gamma$ by deleting the elements having indices $i$ and $i, j$ not belonging to $I$. Also note that (7) has sense only if $\Gamma$ is not singular, i.e., since it is the Gramm matrix, the vectors $\bar{c}_{i}-\bar{c}_{0}$ are linearly independent. As we will see later, it is indeed the case.

Thus we have the following upper bound:

$$
t_{L} \leq \max _{\text {admissible }\left\{\varphi_{i}, \rho_{i}\right\}} \min _{\text {admissible }\left\{\theta_{i}\right\} \text { for }\left\{\varphi_{i}, \rho_{i}\right\}} t\left(d_{0}, \ldots, d_{L-1}\right) .
$$

Since the bound (8) is rather cumbersome, uses complicated optimizations, and is not given in a closed analytical form, we will simplify it. Apparently, the considered simplification essentially worsens the bound (8); however, it allows explicitly demonstrating that it improves on the bound (1) in a low rates range. We restrict our attention to the case where $d_{i}=d_{j}, i, j>1$, and moreover to the situation where at the first step we choose the angle $\theta_{1}$ defined by the equation

$$
R=R_{0}=\min _{\theta / 2 \leq \varphi \leq \pi / 2}\left(R_{\ell}\left(\alpha_{\varphi}(\theta)\right)-\ln \sin \varphi\right)
$$

or

$$
\theta_{1}=\theta_{\ell}^{\prime}(R) \triangleq 2 \min _{e^{-R} \leq \phi \leq \pi / 2} \arcsin \left(\phi \sin \frac{\theta_{\ell}(R+\ln \sin \phi)}{2}\right) .
$$

One can easily see that these formulas are the equations which yield the best known upper bound for the conventional $(L=1)$ packings of the Euclidean sphere [6].

Let us start from a code $\mathcal{K}_{0} \subset \mathbb{S}^{n-1}$ of rate $R=R_{0}$. Let $\varphi_{1}=\varphi$ be the value on which the minimum in (9) is achieved. Set

$$
\rho_{1}=\rho_{\ell}\left(R+\ln \sin \varphi_{0}\right)-\epsilon, \quad \epsilon \ll \rho_{\ell}(R+\ln \sin \varphi) .
$$

The function

$$
f(\theta, \rho)=-2 \ln \sin \varphi+\ln \sin \beta_{\varphi}(\theta)+j\left(\cos \alpha_{\varphi}(\theta), \rho\right)
$$

is increasing in $\theta$, and

$$
f(0, \rho)=(1+\rho) H\left(\frac{\rho}{1+\rho}\right) .
$$

Thus, for some $\theta_{1}<\theta_{\ell}^{\prime}(R)+\delta, \delta \ll \theta_{\ell}^{\prime}$,

$$
R_{1} \geq R-(R+\ln \sin \varphi)-f\left(\theta_{1}, \rho_{1}\right)=\epsilon_{1} .
$$

Next, for the code $\mathcal{K}_{1}^{n} \subset \mathbb{S}^{n-1}\left(\overline{0}, \sin \theta_{\ell}^{\prime}(R)\right)$ of rate $R_{1}$, we use a version of the Plotkin bound, which is a particular case of (1). Actually, in [4] it was proved that for a 
fixed $N$, one can extract a simplex $\bar{c}_{1}, \ldots, \bar{c}_{N}$ from an arbitrary code of growing size on the sphere of a fixed radius such that

$$
d\left(\bar{c}_{i}, \bar{c}_{j}\right)=d+o(1), \quad i \neq j,
$$

i.e., the pairwise distances between vectors from this simplex asymptotically do not depend on the choice of these pairs. We omit the proof of this fact here and refer to the one in [4]. Then (1) yields that $\left\|\bar{c}_{i}-\bar{c}_{j}\right\| \leq \sqrt{2} r$, where $r$ is the radius of the sphere, and in our case,

$$
r=\sin \theta_{\ell}^{\prime}(R)
$$

We have

$$
\left\|\bar{c}_{i}-\bar{c}_{j}\right\| \leq \sqrt{2} \sin \theta_{\ell}^{\prime}(R) .
$$

Note that these last considerations are consistent with the previous procedure where we consequently chose the codes $\mathcal{K}_{1}^{n}, \ldots, \mathcal{K}_{L}^{n}$. Indeed at each step $i \geq 1$ we choose $\mathcal{K}_{i}^{n}$ such that $\left|\mathcal{K}_{i}^{n}\right|=\exp \left(\epsilon_{i} n\right)$ with the minimum distance

$$
d \leq \sqrt{2} \sin \theta_{\ell}^{\prime}(R)
$$

Now we have the simplex $\bar{c}_{0}, \ldots, \bar{c}_{L}$ with $d\left(\bar{c}_{i}, \bar{c}_{j}\right)=d+o(1), i \neq j>0$. As we have already mentioned, the minimum radius $r_{L}$ of a closed ball which contains all these points is determined by (7).

From the theorem of cosines it follows that

$$
\begin{aligned}
d^{2}+o(1) & =\left(\bar{c}_{i}-\bar{c}_{j}\right)^{2} \\
& =\left(\bar{c}_{i}-\bar{c}_{0}\right)^{2}+\left(\bar{c}_{j}-\bar{c}_{0}\right)^{2}-2\left(\bar{c}_{i}-\bar{c}_{0}, \bar{c}_{j}-\bar{c}_{0}\right) \\
& =2 r^{2}-2\left(\bar{c}_{i}-\bar{c}_{0}, \bar{c}_{j}-\bar{c}_{0}\right) ;
\end{aligned}
$$

therefore (up to $o(1)$ ),

$$
\Gamma_{i j}=r^{2}-d^{2} / 2, \quad i \neq j,
$$

and

$$
\Gamma_{i i}=r^{2} .
$$

Here $r$ is the radius of the sphere which is the cross-section of the unit sphere by the hyperplane.

Thus, we have

$$
\Gamma=\left(\begin{array}{cccccc}
a & b & b & b & \ldots & b \\
b & a & b & b & \ldots & b \\
\vdots & & & & \ldots & \\
b & b & b & b & \ldots & a
\end{array}\right)
$$

where

$$
a=r^{2}, \quad b=r^{2}-d^{2} / 2,
$$


$\Gamma^{I}$ has the same form, and $\gamma=(a, a, \ldots, a)$. Furthermore,

$$
\operatorname{det} \Gamma^{I}=(a-b)^{|I|-1}((|I|-1) b+a) .
$$

At the same time,

$$
\operatorname{det} \Gamma^{I}=a A_{i i}^{I}+b \sum_{k: k \neq i} A_{i k}^{I}
$$

and

$$
\begin{aligned}
\sum_{k} A_{i k}^{I} & =A_{i i}^{I}+\sum_{k: k \neq i} A_{i k}^{I} \\
& =A_{i i}^{I}+\frac{1}{b}\left(b \sum_{k: k \neq i} A_{i k}^{I}\right) \\
& =A_{i i}^{I}+\frac{1}{b}\left(b \sum_{k: k \neq i} A_{i k}^{I}+a A_{i i}^{I}-a A_{i i}^{I}\right) \\
& =\frac{1}{b} \operatorname{det} \Gamma^{I}+A_{i i}^{I}\left(1-\frac{a}{b}\right)
\end{aligned}
$$

where $A_{i k}^{I}$ is the algebraic complement of the element $\Gamma_{i k}^{I}$.

Hence,

$$
\begin{aligned}
\gamma^{I}\left(\Gamma^{I}\right)^{-1}\left(\gamma^{I}\right)^{T} & =\frac{a^{2} \sum_{k, i} A_{i, k}^{I}}{\operatorname{det} \Gamma^{I}} \\
& =a^{2} \sum_{k} \frac{\sum_{i} A_{i k}^{I}}{\operatorname{det} \Gamma^{I}} \\
& =\frac{a^{2}|I| \operatorname{det} \Gamma^{I}}{b \operatorname{det} \Gamma^{I}}+\frac{a^{2} A_{i i}^{I}\left(1-\frac{a}{b}\right)|I|}{\operatorname{det} \Gamma^{I}} \\
& =\frac{a^{2}|I|}{b}+\frac{a^{2}|I|(a-b)^{|I|-2}((|I|-2) b+a)\left(1-\frac{a}{b}\right)}{(a-b)^{|I|-1}((|I|-1) b+a)} \\
& =\frac{a^{2}|I|}{b}-\frac{a^{2}|I|((|I|-2) b+a)}{b((|I|-1) b+a)} \\
& =\frac{a^{2}|I|}{(|I|-1) a+b}
\end{aligned}
$$

We see that $\gamma^{I}\left(\Gamma^{I}\right)^{-1}\left(\gamma^{I}\right)^{T}>0$ for all choices of nonempty $I$. Computing the derivative of the expression (11) over $|I|$, we see that it is positive for $a>b$, and hence $\max$ in (7) is attained on $I=\{1,2, \ldots L\}$. Furthermore,

$$
\gamma \Gamma^{-1} \gamma^{T}=\frac{a^{2} L}{(L-1) b+a}
$$


and

$$
t=\frac{a}{2} \sqrt{\frac{L}{(L-1) b+a}} .
$$

Taking into account relation (10), we obtain

$$
\begin{aligned}
t & =\frac{r^{2}}{2} \sqrt{\frac{L}{(L-1)\left(r^{2}-d^{2} / 2\right)+r^{2}}} \\
& =\frac{r}{2}\left(1-\frac{L-1}{2 L} \frac{d^{2}}{r^{2}}\right)^{-1 / 2} .
\end{aligned}
$$

Since $r=2 \sin \frac{\theta_{\ell}^{\prime}(R)}{2}$ and (we omit $o(1)$ )

$$
d=\sqrt{2} \sin \theta_{\ell}^{\prime}(R)=2 \sqrt{2} \sin \frac{\theta_{\ell}^{\prime}(R)}{2} \cos \frac{\theta_{\ell}^{\prime}(R)}{2},
$$

from (12) we get

$$
\begin{aligned}
t & =\frac{\sin \frac{\theta_{\ell}^{\prime}(R)}{2}}{\sqrt{\frac{1}{L} \cos ^{2} \frac{\theta_{\ell}^{\prime}(R)}{2}+\sin ^{2} \frac{\theta_{\ell}^{\prime}(R)}{2}}} \\
& =\left(1+\frac{1}{L} \cot ^{2} \frac{\theta_{\ell}^{\prime}(R)}{2}\right)^{-1 / 2},
\end{aligned}
$$

or

$$
\begin{gathered}
\cot \frac{\theta_{\ell}^{\prime}(R)}{2}=\sqrt{L t^{-2}-L,}, \\
\theta_{\ell}^{\prime}(R)=2 \operatorname{arccot} \sqrt{L t^{-2}-L} .
\end{gathered}
$$

Now using (9) we obtain the following bound:

$$
\begin{aligned}
& 2 \operatorname{arccot} \sqrt{L t^{-2}-L} \\
& \quad \leq 2 \min _{\arcsin e^{-R} \leq \varphi \leq \pi / 2} \arcsin \left(\sin \varphi \sin \frac{\theta_{\ell}(R+\ln \sin \varphi)}{2}\right) .
\end{aligned}
$$

Denote

$$
q=1 / \min _{e^{-R} \leq \phi \leq 1}\left(\phi \sin \frac{\theta_{\ell}(R+\ln \phi)}{2}\right) .
$$

Then (13) is equivalent to the inequality, which is our final bound,

$$
t_{L} \leq\left(\frac{L-1}{L}+q^{2} / L\right)^{-1 / 2} .
$$


Theorem 2 is proved.

One can easily see that the derivative in $R$ of the right-hand side of (14) is

$$
\frac{2 q q_{R}^{\prime}}{L\left(q^{2} / L+(L-1) / L\right)^{3 / 2}} \stackrel{R \rightarrow 0}{\rightarrow}-\infty,
$$

and at the same time, the derivative in $R$ of the right-hand side of

$$
t_{L} \leq \sqrt{\frac{L}{L+1}} e^{-R},
$$

equals $-\sqrt{\frac{L}{L+1}}$ for $R=0$. Moreover, they both equal $\sqrt{\frac{L}{L+1}}$ at the zero rate. This proves that the bound (14) is better on some interval of low rates.

\section{References}

1. Ashikhmin, A., Barg, A., Litsyn, S.: A new upper bound on the reliability function of the Gaussian channel. IEEE Trans. Inf. Theory 46(6), 1945-1961 (2000)

2. Ben-Haim, Y., Litsyn, S.: Improved upper bounds on the reliability function of Gaussian channel. IEEE Trans. Inf. Theory 54(1), 5-12 (2008)

3. Blachman, N., Few, L.: Multiple packing of spherical caps. Mathematika 10, 84-88 (1963)

4. Blinovsky, V.: Multiple packing of the Euclidean sphere. IEEE Trans. Inf. Theory 45(4), 1334-1337 (1999)

5. Bocharova, I., Johannesson, R., Kudryashov, B., Loncar, M.: An improved bound on the list error probability and list distance properties. IEEE Trans. Inf. Theory 54(1), 13-32 (2008)

6. Kabatyansky, G., Levenshtein, V.: Bounds for packing on the sphere and in the space. Probl. Inf. Transm. 14(1), 3-25 (1978) 